\title{
Improvement of Cleaning Method in Spinning Mills
}

\author{
Part 3: Cleaning Characteristics of Blow Nozzle
}

\section{By Yasushi Niitsu, ${ }^{*}$ Shigeki Fujimori** and Yoshinobu Hosokawa** Members, TMSJ}

* Faculty of Engineering, Osaka University, Osaka.

** Himeji Technical College, Himeji, Hyogo Pref.

\begin{abstract}
A bstract
Object of study :

Use of jet flow is a conceivable method to remove fly, cotton tufts, dust and the like which scatter in a vast area of a spinning mill, but data on it are lacking. We have made experiments by discharging air flow from a circular nozzle located above a plane in an effort to investigate the nature and characteristics of air flow and obtain data which will aid in the designing of a blow nozzle for an improved cleaning method for spinning mills.
\end{abstract}

Results :

1. Theoretical formulas have been obtained on the basis of Reichardt's hypothesis as to free circular jet, a radial jet and a reciprocal interference between two parallel jets.

2. The reasonableness of our formulas has been proved experimentally and values for constant $C$ in Reichardt's theory determined. Further, we have derived, for practical use, approximate formulas on the air velocity distribution based on the theoretical formulas mentioned above.

3. We have measured the velocity distribution, an important factor in dust-removing, and determined the most effective conditions for the location of the nozzle:

\section{Introduction}

To improve the cleaning method in spinning mills, it would be wise to use the sucking method and the blowing method together, not separately.

In two previous articles we tried to explain, theoretically and experimentally, the mechanism of the cleaning method by sucking and obtained adequate data on the designing of a sucking nozzle. In the present article we raport the results of our study on cleaning by jet flow.

There are many fluid-dynamical studies published on gas jet which issues from a nozzle into a stationary atmosphere, but few, if any, studies about a jet which strikes a plane surface before diverging. We have, therefore, studied the nature and characteristics of a jet against a plane surface from the fluid-dynamical point of view, so that we can find a way to remove accumulated dust from a plane surface and obtain data which will aid in the designing of a blowing nozzle. 
1. Nature and the Characteristics of a Jet Discharged Vertically Against a Plane Surface

\section{1-1. Theoretical analysis}

Jet flow vertical to a plane has forms which can be classified roughly into two types, (a) and (b) shown in Fig. 1. (a) is the form where the plane is close to the nozzle and the potential core of the jet reaches the plane. (b) is the form where the plane is far from the nozzle and the plane is under the influence of a fully developed flow of jet. In either case, jet flow itself is devided into three parts :

1) Region $\mathrm{A}$ in which air flow may be considered a free circular jet.

2) Region $\mathrm{C}$ in which air flow can be considered a radial jet.

3) Region B in which air flow takes neither the form of a free circular jet nor the form of a radial jet, but takes an intermediate form between these two and is under the influence of the plane.

In this study jet which dashes against, and is influenced by, the plane is considered to be a combination of a free jet and a radial jet.

The authors based their theoretical analysis on the following hypothesis by Reichard[1]:

(a) That the momentum of a free jet is almost completely preservable.

(b) That the distribution of the flux momentum as a function of the distance from the jet axis can be expressed approximately by the Gaussian distribution.

(c) That a free jet spreads with a nearly constant angle of divergence.

First, about a free circular jet. Take an axial

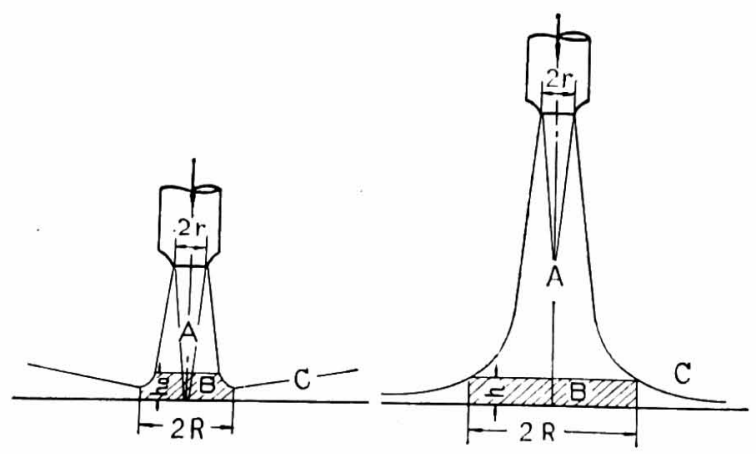

(a)

Fig. 1 Jet flow against a plane symmetrical jet issuing from small outlet, assume an $r, \theta, z$ cylindrical co-ordinate ( $z$ denoting the flow direction) with the outlet as the origin, and assume that the velocity in direction $z$ is $u$ and the velocity in direction $r$ is $v$. We can then obtain eq. (1) derived from the equation of motion and that of continuity

$$
\frac{\partial u^{2}}{\partial z}+\frac{1}{r} \frac{\partial}{\partial r}(r u v)=0
$$

where the effect of pressure and viscosity is neglected and density $\rho$ of gas is assumed to be constant.

Reichardt writes empirically

$$
u v=-\wedge \frac{\partial u^{2}}{\partial r}
$$

Assuming the momentum transfer length to be of the form

$$
\wedge=\left(\frac{b}{2}\right)\left(\frac{d b}{d z}\right)
$$

eq. (1) transforms itself thus:

$$
\frac{\partial u^{2}}{\partial z}-\frac{b}{2 r}\left(\frac{d b}{d z}\right) \frac{\partial}{\partial r}\left(r \frac{\partial u^{2}}{\partial r}\right)=0
$$

From assumptions (a), (b) and (c) a particular solution of differential eq. (3) emerges:

$$
u^{2}=\frac{u_{0}^{2} F_{0}}{\pi c^{2} z^{2}} \mathrm{e}^{-(r / c z)^{2}}
$$

Here $c$ is a constant, $u_{0}(\mathrm{~m} / \mathrm{s})$ is the velocity at the outlet and $F_{0}\left(\mathrm{~m}^{2}\right)$ is the area of the outlet.

Eq. (3) being a linear differential equation, the principle of superposition holds on the particular solution (4). Assuming $r_{0}$ to be the radius of the outlet, then:

$$
\left(\frac{u}{u_{0}}\right)^{2}=1-e^{-\left(r^{2}+r_{\|^{2}} 2 c^{2} z^{2}\right)} \sum_{p=0}^{\infty}\left(\frac{r}{r_{0}}\right)^{p} \frac{J_{p}\left(\frac{2 i r r_{0}}{c^{2} z^{2}}\right)}{(i) p}
$$

On the jet axis

$$
\frac{u_{c}}{u_{0}}=\sqrt{1-e^{-\left(r_{0} / c z\right) 2}}
$$

Since $c z \ll r_{0}$ in the vicinity of the source,

$$
\begin{aligned}
& \left(\frac{u}{u_{0}}\right)^{2} \fallingdotseq \frac{1}{6}\left(6 e^{-(r / c z) 2}-e^{-\left(r-r_{0} / c z\right) 2}\right. \\
& \left.+\frac{r}{c z} \sqrt{\pi}\left\{2 \phi\left(\frac{r}{c z}\right)-\phi\left(\frac{r-r_{0}}{c z}\right)\right\}\right)
\end{aligned}
$$

where $\phi(\xi)=\frac{2}{\sqrt{\pi}} \int_{0}^{\xi} e^{-x^{2}} d t=$ probability integral

Since, far from the source, $r_{0} \ll c z$,

$$
\frac{u}{u_{0}}=\left(\frac{r_{0}}{c z}\right) e^{-1 / 2(r / c z) 2}
$$


Now about a radial jet. Take a radial jet discharged from the slit of a circular ring $R$, in radius and $2 h_{0}$ in width and assume an $r, \theta, z$ cylindrical co-ordinate ( $r$ being the flow direction) which takes the center of the circular ring as its original, as shown in Fig. 2. We can then deduce the following equation, as in the case of a free circular jet :

$$
\frac{\partial u^{2}}{\partial r}+\frac{u^{2}}{r}+\frac{\partial}{\partial z}(u v)=0
$$

where the velocities of $r, z, \theta$-direction are respectively $u(m / s), v(m / s)$, and zero.

A particular solution of eq. (9), then, is :

$$
u^{2}=\frac{u_{R}^{2} F_{0}}{2 \pi \sqrt{\pi} C_{r}(r-R)} e^{-\{z / c(r-R)\}^{2}}
$$

Here $u_{R}(m / s)$ is the jet velocity at the slit and its distribution over the width of $2 h_{0}$ is uniform all along. Eq. (9) being a linear differential equation, we can superpose its solution (10). Hence:

$$
\frac{u}{u_{R}}=\sqrt{\frac{1}{2} \frac{R}{r}\left[\phi\left\{\frac{z+h_{0}}{c(r-R)}\right\}-\phi\left\{\frac{z-h_{0}}{c(r-R)}\right\}\right)}
$$

Since $c(r-R) \ll h_{0}$ in the vicinity of the source,

$$
\frac{u}{u_{R}} \doteqdot \sqrt{-\frac{1}{2}} \frac{R}{r}\left(1-\phi\left\{\frac{z-h_{0}}{c(r-R)}\right\}\right)
$$

Since, far from the source, $c(r-R) \gg h_{0}$,

$$
\frac{u}{u_{R}} \doteqdot \sqrt{\frac{R}{r} \quad c(r-R) \sqrt{\pi}} e^{-1 / 2(z / c(r-R))^{2}}
$$

On the jet axis, substituting $z=0$ into eq. (11) gives :

$$
u_{u}=\sqrt{ } \frac{R}{r} \phi\left\{\frac{h_{0}}{c(r-R)}\right\}
$$

The above equations are adequate if a jet flow retains the condition described in Fig. 1(a). However,

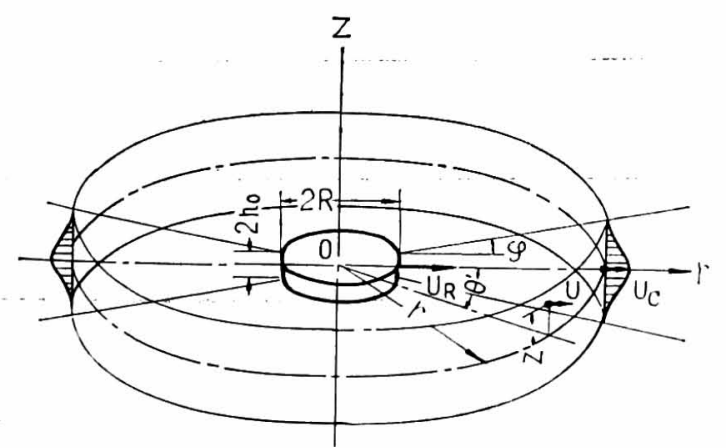

Fig. 2 Radial jet discharged from the slit of a circular ring as shown in Fig. 1(b), if a jet strikes the plane with its fully developed flow, the value of $\varkappa_{R}$ becomes a function of $z$, since the width of the slit is much larger than that of Fig. 1(a) and the value of $u_{k}$ cannot be considered uniform over the whole width of the slit,

Therefore, by expressing $u_{R}(z)$ as a function of $z$, in which $u_{R}$ becomes $u_{R 0}$ at $z=0, u_{R}=0$ at $z= \pm h_{0}$, and by applying Simpson's approximate intergral, we get:

$$
\frac{u}{u_{R 0}} \doteqdot 0.816 \sqrt{\frac{1}{2}} \frac{R}{r}\left[\phi\left\{\frac{z+h_{0}}{c(r-R)}\right\}-\phi\left\{\frac{z-h_{0}}{c(r-R)}\right\}\right)
$$

\section{1-2. Determination of constant $c$ in Reichardt's theory}

To investigate the characteristics of a circular jet striking the plane vertically, we mounted $30 \mathrm{~mm} \phi$, or $20 \mathrm{~mm}^{\mathrm{p}}$ standard nozzle on one end of a 2 -in steel pipe vertically against the plane, and measured the air velocity in each section normal to the jet axis. We then measured the velocity in each section vertical to the plane with a calibrated pitot tube, $2 \mathrm{~mm}$ in outside diameter and $1 \mathrm{~mm}$ in inside diameter, mounted on a differential moving device and connected to Göttingen manometer.

The values of $c$ in the above formulas are constants determinable only by experiment. To determine the $c$-values for a free circular jet, we measured the values of $u_{c}$ and applied eq.(6) with the following result:

$$
1 \frac{1}{\sqrt{-l n}\left\{1-\left(\frac{u_{c}}{u_{0}}\right)^{2}\right\}}=\frac{z}{r_{0}}
$$

Hence we can draw a straight line as shown in Fig. 3 and get the value 0.08[2] of $c$ from the tangent of the line. The $c$-value of the radial flow varies according to the relative position of the plane. Therefore, by putting

$$
\frac{r}{R}\left(\frac{u_{c}}{u_{R}}\right)^{2}=\phi(\lambda)
$$

into eq. (14) for regions close to the plane, we get:

$$
\frac{h_{0}}{r-R}=c \lambda
$$

From eq. (17) and Fig. 4, then, we get $c=0.16$.

For regions close to a jet source, substituting $1-2\left(\frac{u}{u_{c}}\right)^{2}=\phi(\lambda)$ into eq. (12) gives us : 


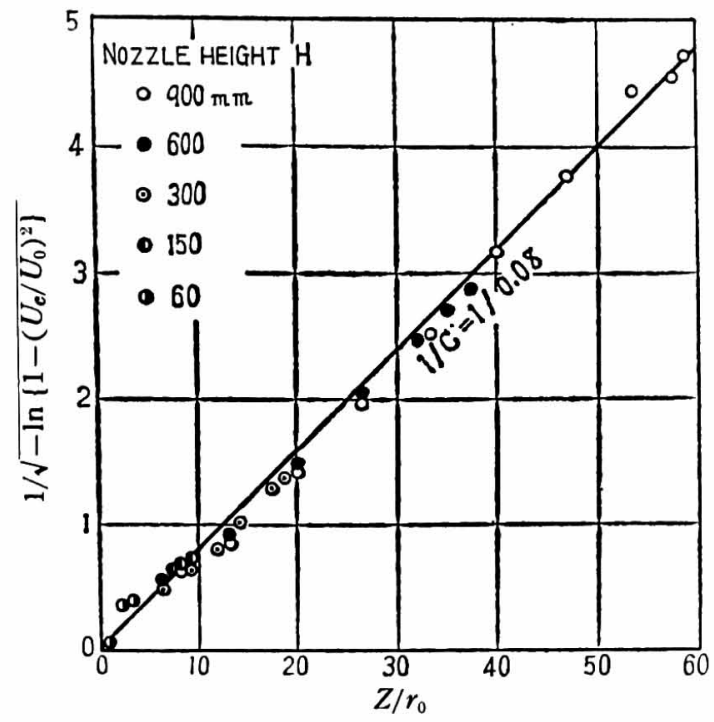

Fig. 3 Derivation of constant $c$ when circular jet strikes a plane vertically

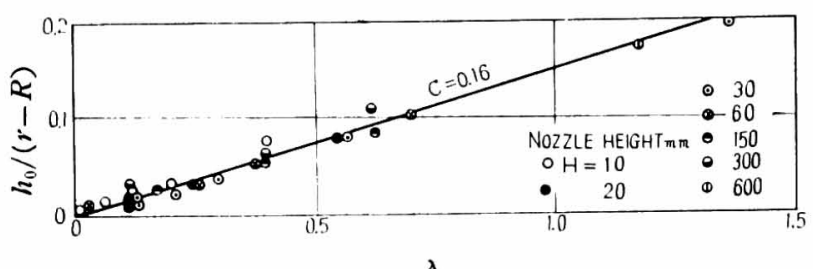

Fig. 4 Derivation of constant $c$ for the radial flow in the vicinity of the plane

$$
\frac{z-h_{0}}{r-R}=c \lambda
$$

From Fig. 5, $c$ is determined to be 0.08 .

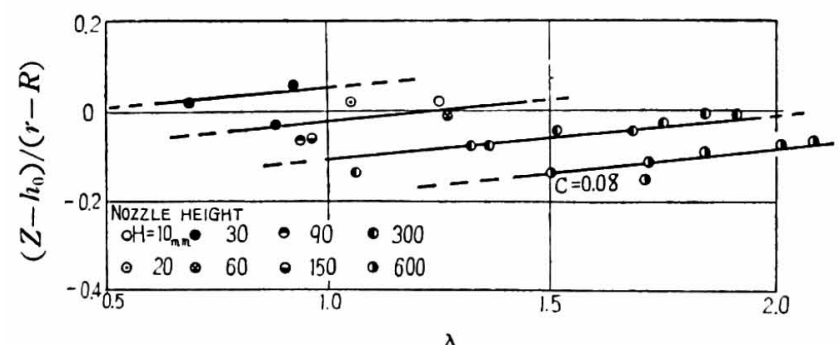

Fig. 5 Constant $c$ for the radial flow in the vicinity of the jet sourse

Lastly, for regions far from the source:

$$
-l_{n}\left(\frac{u}{u_{c}}\right)=\frac{1}{2}\left\{\frac{z}{r-R}\right\}^{2} \frac{1}{C^{2}}
$$

From Fig. 6, $c$ is determined to be 0.08 .

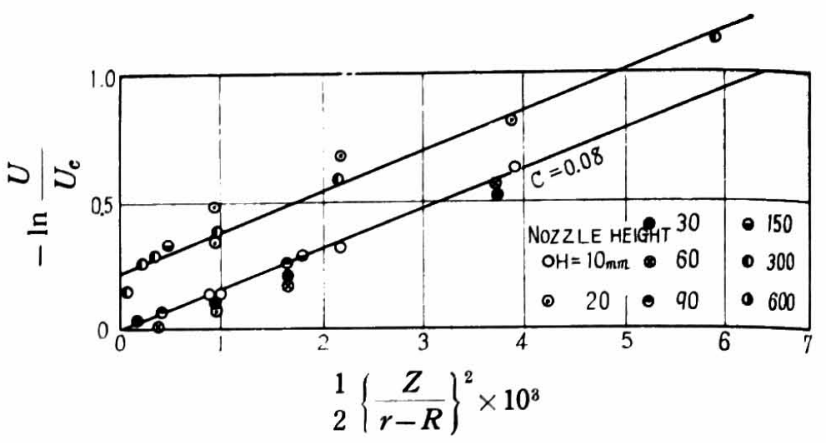

Fig. 6 Constant $c$ for the radial flow in regions for from the source

1-3. Regions under the influence of the plance

The nature of a free jet changes in regions close to the plane. We, therefore, investigated experimentally a substantial part of a free jet and a radial jet.

It is believed that constant $c$ in the foregoing equations vary as it gets closer to the plane. A linear relation is obtained between $1 / \sqrt{-l_{n}}\left\{1-\left(u_{c} / u_{0}\right)^{2}\right]$ and $z$ on eq. (16). Giving $c$ in the form of its tangent $r_{0} / c, c$ for that region of air flow which is not considered a free jet can thus be obtained. This relationship is shown in Fig. 7. A free jet is influenced by the floor in the region $40 \sim 50 \mathrm{~mm}$ from the plane, no matter what the nozzle height is.

We measured the pressure on the floor to see what the range of a radial jet was. Pressure $p_{0,}$ naturally, has a maximum value directly under the

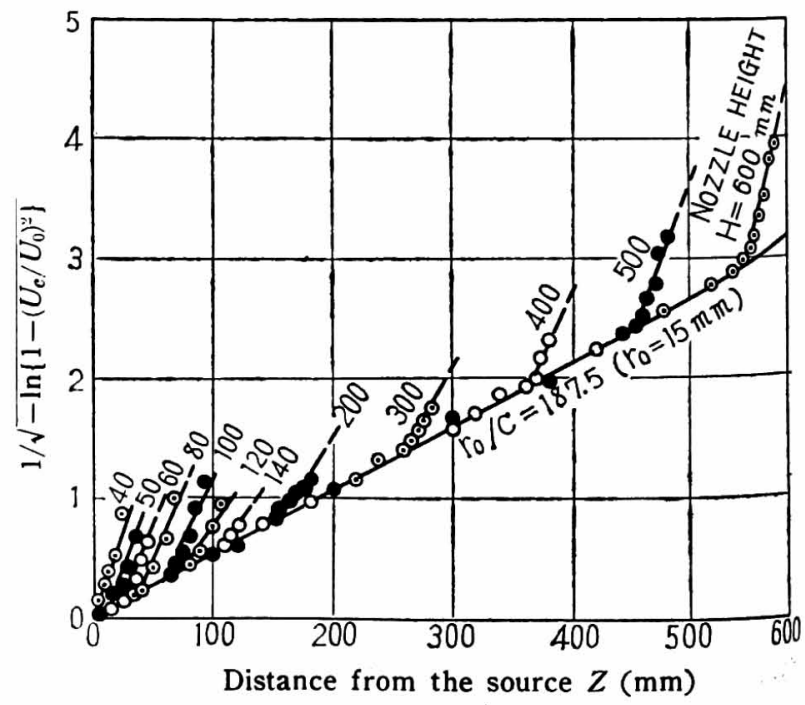

Fig. 7 Regions under the influence of the plane 
nozzle and makes a steep decline just outside the circle (distance $R$ from the center) where the radial jet begins. Therefore, the distribution of $p / p_{0}$ becomes as shown in Fig. 8 , in which the location of the above circles is given as an inflexion point, and the values of $R$ are

$$
\begin{array}{ll}
R=2.45 r_{0} & \left(H \leqq 7 r_{0}\right) \\
R / r_{0}=H / 7 r_{0}+1.7 & \left(H \geqq 7 r_{0}\right)
\end{array}
$$

The relation between the height of a jet layer and the nozzle height in the region of a radial jet is shown in Fig. 9, the height $h_{0}$ being

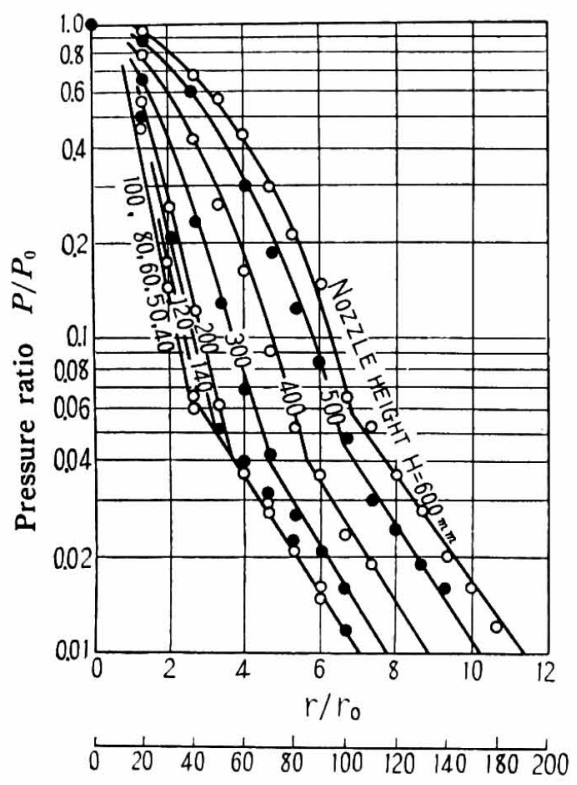

Distance from the nozzle center $r(\mathrm{~mm})$

Fig. 8 Pressure distribution on the floor

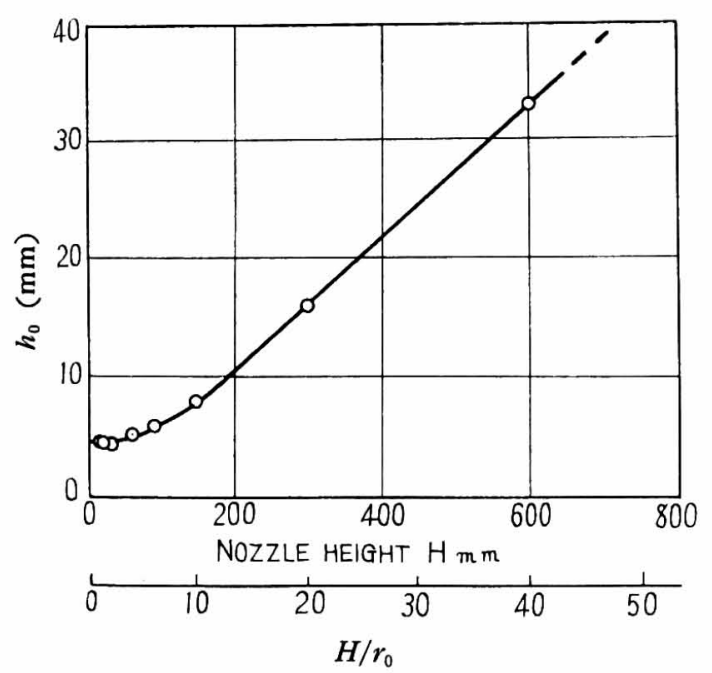

Fig. 9 Relation between the height of a jet layer and the nozzle height in the region of a radial jet

$$
\begin{array}{ll}
h_{0}=5 & \left(H \leqq 3 r_{0}\right) \\
h_{0}=0.83\left(H / r_{0}\right)-0.40 & \left(H \geqq 10 r_{0}\right)
\end{array}
$$

\section{1-4. Experimental results}

Fig. 10 gives the theoretical and experimental values of air velocity for a nozzle height of $20 \mathrm{~mm}$, where the potential core of a free circular jet strikes the plane and scatters. Fig. 11 gives such values for a nozzle height of $600 \mathrm{~mm}$, where the fully developed flow of a jet strikes the plane.

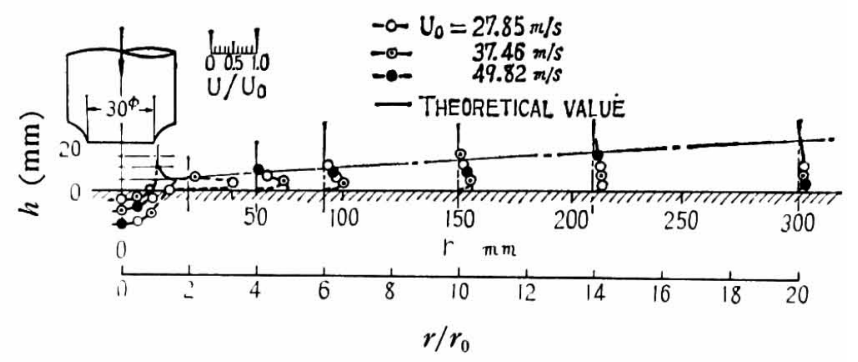

Fig. 10 Theoretical and experimental values of velocity for a nozzle height $20 \mathrm{~mm}$

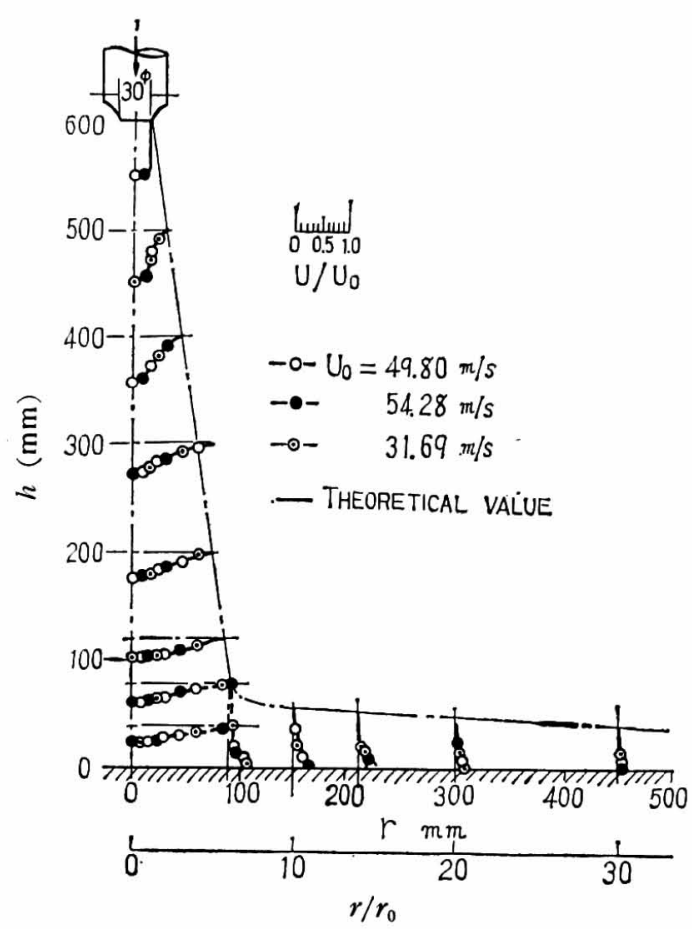

Fig. 11 Theoretical and experimental values of velocity for a nozzle height $600 \mathrm{~mm}$ 
The velocity distribution, if expressed as the ratio $u / u_{0}$, shows the same distribution, regardless of the magnitude of $u_{0}$.

Jets from nozzles of various heights photographed by the smoke technique are shown in Fig. 12 .

Assuming that the outer boundary of the jet is defined as the place where the air speed is $5 \%$ of that at the core, then the angle of divergence for a free circular jet is about $20^{\circ}$ and the same angle is $10^{\circ}$ if the nozzle is close to the plane. The angle of divergence decreases as the nozzle gets further away from the plane. The authors confirmed this with the aid of photographs. These values are approximately equal to those obtained by other workers.[3]
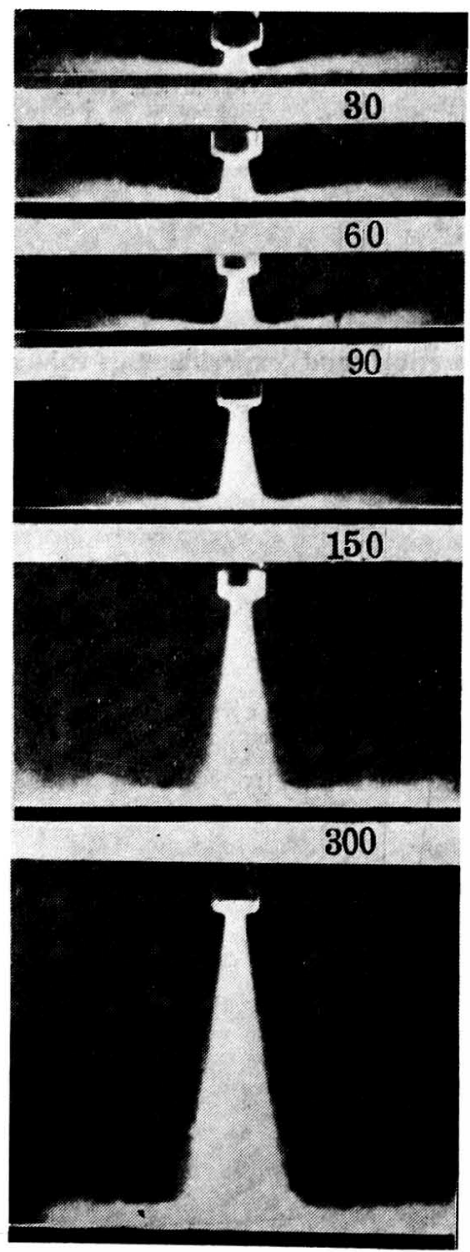

Nozzle hight $450(\mathrm{~mm})$

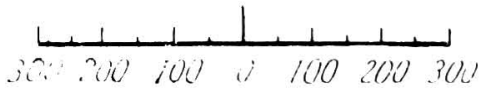

The distance from the nozzle center ( $\mathrm{mm}$ )

Fig. 12 Jets from nozzles of various heights
The relation between the velocity ratio $u_{c} / u_{0}$ on the jet axis and $(H-h+r) / r_{0}$ is as shown in Fig. 13, where we chose the velocity at a $2.5 \mathrm{~mm}$ level from the floor as $u_{0}$ of a radial jet, which level was just above the boundary layer. The arrows in Fig. 13 show the positions of the floor, the full lines give the theoretical values of the velocity ratio and the dotted lines give those of section B in Fig. 1 . Therefore, $u_{c} / u_{0}$ of a free circular jet is as follows:

$$
\begin{aligned}
& u_{c} / u_{0}=1 \quad\left(z \leqq 12 r_{0}\right) \\
& u_{c} / u_{0}=10\left(z / r_{0}\right)^{-0.95} \quad\left(z \geqq 12 r_{0}\right)
\end{aligned}
$$

The maximum value of air velocity in the region of a radial jet is approximately given as follows:

where

$$
\begin{gathered}
u_{c} / u_{0}=e^{k}\left(H-h+r / r_{0}\right)^{-k} \\
k=0.37\left(H / r_{0}\right)+1.30
\end{gathered}
$$

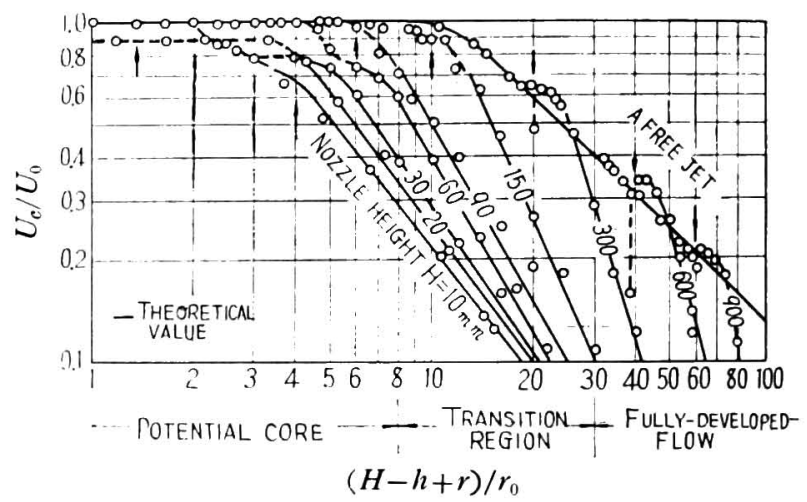

Fig. 13 Relation between velocity ratio $u_{c} / u_{0}$ on the jet axis and $(H-h+r) / r_{0}$

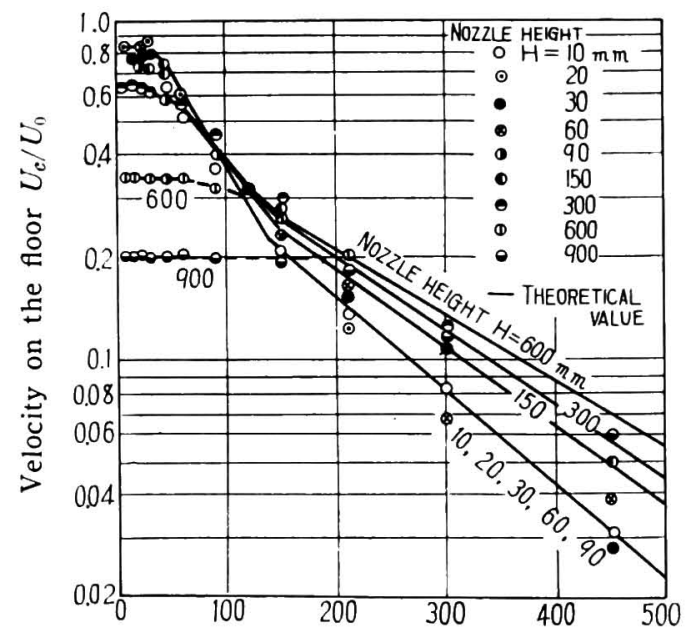

The distance from the nozzle center $r(\mathrm{~mm})$

Fig. 14 Velocity distribution in layer $2.5 \mathrm{~mm}$ high on the floor 


$$
\begin{array}{ll}
K=1.3\left(H / r_{0}\right)^{0.2} & \left(H \leqq 7 r_{0}\right) \\
K=0.8\left(H / r_{0}\right)^{0.5} & \left(H \geqq 7 r_{0}\right)
\end{array}
$$

The air flow along the floor plays a principal part in blowing off fly, cotton tufts, dust, etc. accumulated on the plane. Fig. 14 shows the velocity distribution along a layer $2.5 \mathrm{~mm}$ above the floor. When the nozzle is close to the plane and the potential core of a jet strikes the plane, the jet is not suitable for removing light-weight dust because the angle of divergence of the jet is large and the stream lines bounce back. Accordingly, the effective height for cleaning is

$$
H=20 d_{0} \sin \theta
$$

\section{Characteristics of a Jet Which Is Set Paralell to the Plane and Which Interferes with the Plane}

\section{2-1. Theoretical analysis}

A jet parallel to the plane can be divided into two forms. One form is made where a circular outlet is located fully above the plane as shown in Fig. 15(a). The other form is made where a circle only partially exposed above the plane and is a jet issues from it in the manner shown in Fig. 16(a). Assuming the floor to be the plane of symmetry, the first form results in the reciprocal interference of two free jets[4] as in Fig. 15(b), while the second form is presumably a jet issuing from an outlet of the shape shown in Fig. 16(b).

As in the previous section, we assume that Reichardt's hypothesis holds good here, too. As shown in Figs. 15 and 16(b), we take an $x, y, z$ ( $z$ being the flow direction) rectangular co-ordinate which has, as its origin, the center of the chord formed by the plane and the outlet. Assume $w, v, u(m / s)$ to be the velocities of $x, y, z$-directions, respectively, neglect pressure and viscous effects, assume a uniform density and a jet issuing from an incremental outlet $d x \times d y$ which may be regarded as one point. Then, the following equation is obtainable from the equation of motion and continuity:

$$
\frac{\partial(w u)}{\partial x}+\frac{\partial(v u)}{\partial y}+\frac{\partial u^{2}}{\partial z}=0
$$

Making the same hypothesis as in the foregoing section for the products $v w, v u$ gives us:

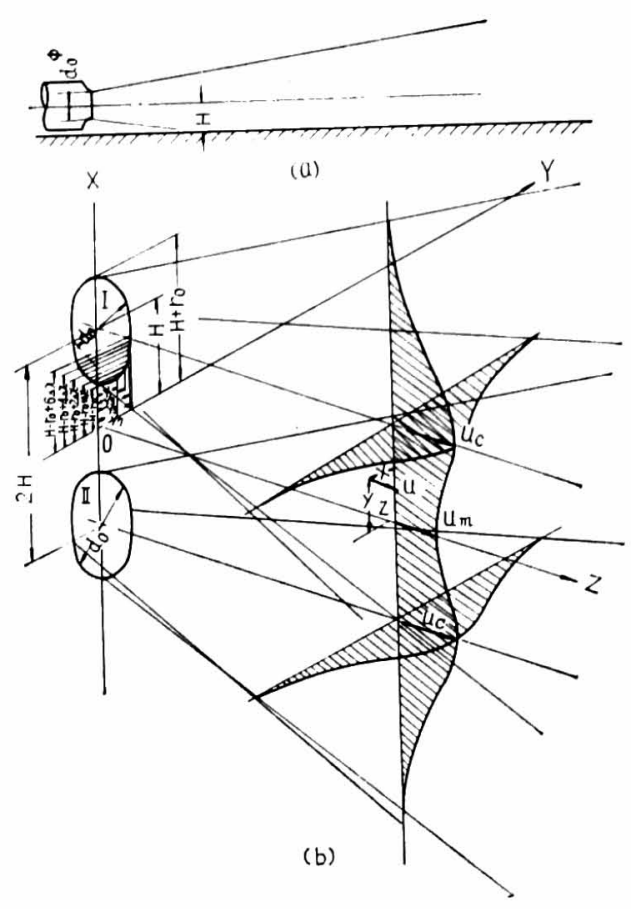

Fig. 15 A jet parallel to the plane when a circular outlet is fully located above the plane

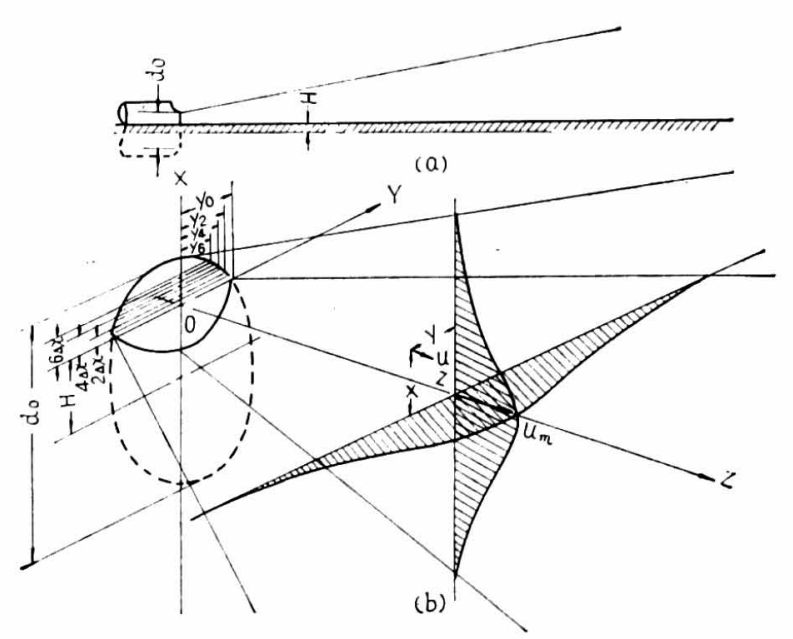

Fig. 16 A jet parallel to the plane when only a part of a circle is exposed on the plane

$$
\frac{\partial u^{2}}{\partial z}-\frac{b}{2} \frac{d b}{d z}\left(\frac{\partial^{2} u^{2}}{\partial x^{2}}+\frac{\partial^{2} u^{2}}{\partial y^{2}}\right)=0
$$

and one of its particular solutions is

$$
u^{2}=\frac{u_{0}^{2} F_{0}}{(c z)^{2} \pi} e^{-\frac{x^{2}+y^{2}}{(c z)^{2}}}
$$

If we assume the outlet to be the collective of incremental rectangles as shown Fig. 16, its approximate solution is 
54 January 1963

$$
\begin{aligned}
& \left(\frac{u}{u_{0}}\right)^{2}=2-e^{-\frac{y^{2}+r_{0}{ }^{2}}{(c z)^{2}}}\left(e^{-\left(\frac{x-H}{c z}\right)^{2}} \sum_{P=0}^{\infty}\left(\frac{\sqrt{(x-H)^{2}+y^{2}}}{r_{0}}\right)^{P} \frac{J_{p}\left(\frac{2 i \sqrt{(x-H)^{2}+y^{2} r_{0}}}{c^{2} z^{2}}\right)}{(i)^{p}}\right. \\
& \left.+e^{-\left(\frac{x+H}{c z}\right)^{2}} \sum_{p=0}^{\infty}\left(\frac{\sqrt{(x+H)^{3}+y^{2}}}{r^{0}}\right)^{p} \frac{J_{p}\left(\frac{2 i \sqrt{(x+H)^{2}+y^{2} r_{0}}}{c^{2} z^{2}}\right)}{(i)^{p}}\right)
\end{aligned}
$$

The velocity $u_{m}(m / s)$ on $z$-axis, with $x=0, y=0$ put into (23), is:

$$
\left(\frac{u_{m}}{u_{0}}\right)^{2}=2-2 e^{-\frac{r_{0}{ }^{2}+H^{2}}{(c z)^{2}}} \sum_{p=0}^{\infty}\left(\frac{H}{r_{0}}\right)^{p} \frac{J_{p}\left(\frac{2 i H r_{0}}{c^{2} z^{2}}\right)}{(i)^{p}}
$$

Since, close to the source, $c z \ll r_{0}$, we obtain from (7)

$$
\begin{aligned}
& \left(\frac{u}{u_{0}}\right)^{2} \fallingdotseq \frac{1}{6}\left[6 e^{-\left(\frac{y}{c z}\right)^{2}}\left\{e^{-\left(\frac{x-H}{c z}\right)^{2}}+e^{-\left(\frac{x+H}{c z}\right)^{2}}\right\}-e^{-\left\{\frac{\sqrt{(x-H)^{2}+y^{2}}-r_{0}}{c z}\right\}^{2}}-e^{-\left\{\frac{\sqrt{(x+H)^{2}+y^{2}}-r_{0}}{c z}\right\}^{2}}+\right. \\
& \frac{\sqrt{(x-H)^{2}+y^{2}}}{c z} \sqrt{\pi}\left\{2 \phi\left(\frac{\sqrt{(x-H)^{2}+y^{2}}}{c z}\right)-\phi\left(\frac{\sqrt{(x-H)^{2}+y^{2}}-r_{0}}{c z}\right)\right\}+\frac{\sqrt{(x+H)^{2}+y^{2}}}{c z} \sqrt{\pi}\left\{2 \phi\left(\frac{\sqrt{(x+H)^{2}+y^{2}}}{c z}\right)\right. \\
& \left.\left.-\phi\left(\frac{\sqrt{(x+H)^{2}+y^{2}}-r_{0}}{c z}\right)\right\}\right]
\end{aligned}
$$

Since, far from the source, $c z \gg r_{0}$, we obtain from (8)

$$
\left(\frac{u}{u_{0}}\right)^{2}=2\left(\frac{r_{0}}{c z}\right)^{2} e^{-\frac{x^{2}+y^{2}+H^{2}}{(c z)^{2}}} \cosh \frac{2 H x}{(c z)^{2}}
$$

Likewise, if the form of the outlet is as in Fig. 16

$$
\begin{aligned}
& \left(\frac{u}{u_{0}}\right)^{2}=\frac{1}{4}\left(\sum_{i=2}^{2 n}\left\{\phi\left(\frac{x-i-1 \Delta x}{c z}\right)-\phi\left(\frac{x-i+1 \Delta x}{c z}\right)+\phi\left(\frac{x+\overline{i+1} \Delta x}{c z}\right)-\phi\left(\frac{x+\overline{i-1} \Delta x}{c z}\right)\right\}\right. \\
& \left.\times\left\{\phi\left(\frac{y+y_{i}}{c z}\right)-\phi\left(\frac{y-y_{i}}{c z}\right)\right\}+\left\{\phi\left(\frac{x+\Delta x}{c z}\right)-\phi\left(\frac{x-\Delta x}{c z}\right)\right\}\left\{\phi\left(\frac{y+y_{0}}{c z}\right)-\phi\left(\frac{y-y_{0}}{c z}\right)\right\}\right]
\end{aligned}
$$

Here $i=2,4,6$ $.2 n$

$$
y_{t}=\sqrt{r_{0}^{2}-\left(r_{0}-x_{0}+i \Delta x\right)^{2}}
$$

The velocity on $z$-axis, with $x=0, y=0$ put into (27), is :

$$
\left(\frac{u_{m}}{u_{0}}\right)^{2}=\sum_{i=2}^{2 n}\left(\phi\left(\frac{\pi+1 \Delta x}{c z}\right)-\phi\left(\frac{i-1 \Delta x}{c z}\right)\right) \phi\left(\begin{array}{c}
y_{l} \\
c z
\end{array}\right)+\phi\left(\frac{\Delta x}{c z}\right) \phi\left(\frac{y_{0}}{c z}\right)
$$

Close to the source

$$
\left(\frac{u}{u_{0}}\right)^{2} \doteqdot \frac{1}{4}\left(\sum_{i=2}^{2 n}\left\{\phi\left(\frac{x-\overline{i-1} \Delta x}{c z}\right)-\phi\left(\frac{x-\overline{i+1} \Delta x}{c z}\right)\right\}\left\{1-\phi\left(\frac{y-y_{i}}{c z}\right)\right\}+\left\{1-\phi\left(\frac{x-\Delta x}{c z}\right)\right\}\left\{1-\phi\left(\frac{y-y_{0}}{c z}\right)\right\}\right)
$$

For from the source,

$$
\left(\frac{u}{u_{0}}\right)^{2} \doteqdot \frac{2}{\pi}\left(\frac{r_{0}}{c z}\right)^{2} \times\left\{\frac{\pi}{2}+\sin ^{-1} \frac{H}{r_{0}}+\frac{y_{0} H}{r_{0}^{2}}\right\} \times e^{-\frac{x^{2}+y^{2}+H^{2}}{(c z)^{2}}} \cosh \frac{2 x H}{(c z)^{2}}
$$


2-2. Determination of constant $c$ in Reichardt's theory

$c$ in the above formulas is a constant which can be determined experimentally. The velocity, far from the source, in the section containing the jet axis and normal to the plane, i.e., in the section of $y=0$, is obtained by the following equation derived from (26) and (30):

$$
\left(\frac{u}{u_{0}}\right)^{2}=\frac{2 k}{\pi}\left(\frac{r_{0}}{c z}\right)^{2} e^{-\frac{x^{2}+H^{2}}{(c z)^{2}}} \cosh \frac{2 x H}{(c z)^{2}}
$$

If a circular outlet appears fully above the plane, $K$ becomes

$$
K=\pi
$$

If only a part of a circular outlet appears above the plane,

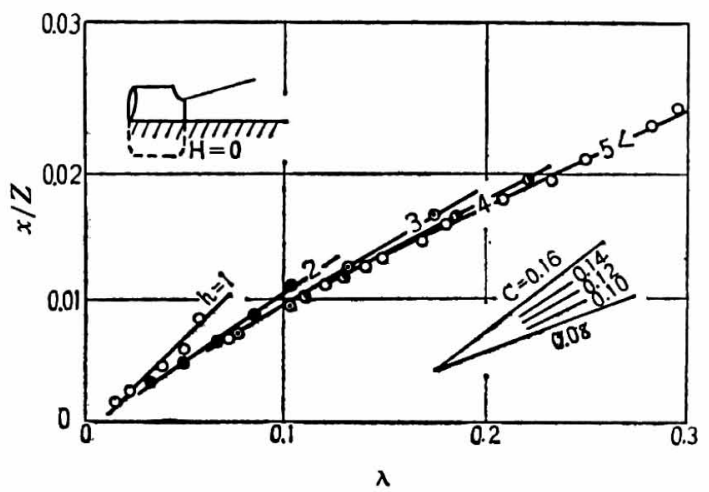

Fig. 17 Constant $c$ of a circular jet parallel to the plane

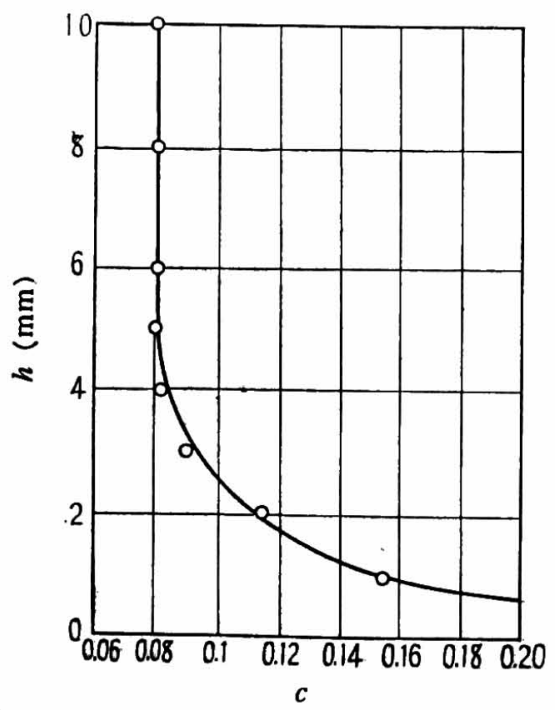

Fig. 18 Constant $c$ of a circular jet parallel to the plane

$$
K=\frac{\pi}{2}+\sin ^{-1} \frac{H}{r_{0}}+\frac{y_{0} H}{r_{0}^{2}}, \quad\left(y_{0}=\sqrt{r_{0}^{2}-H^{2}},\right.
$$

Putting $x / c z=\lambda$ into (31) gives us:

$$
2 \pi\left(\frac{u}{u_{0}} \cdot \frac{x}{r_{0}}\right)^{2}=\lambda^{2} e^{-\lambda^{2}\left\{1+\left(\frac{H}{x}\right)^{2}\right\}} \cosh 2 \lambda^{2} \frac{H}{x}
$$

After the left-side value of the above equation is obtained by experiment, then $\lambda$ corresponding to it is calculable. Hence tangent $c$ can be obtained from the following:

$$
x / z=c \lambda
$$

Examples of the values of $c$ thus obtained are given in Fig. 17. From these figures we find that the value of $c$ is 0.08 at a height of $5 \mathrm{~mm}$ or more from the plane but that the value increases as the nozzle gets closer to the plane as shown in Fig. 18.

\section{2-3. Experimental results}

One of the examples of the experimental and theoretical values of air velocity for a circular jet parallel to the plane is given in Fig. 19. Fig. 20 shows the state of a jet along the cross section containing the nozzle axis and normal to the plane. From the photographs we see that the angle of divergence of the jet is $10^{\circ}$, assuming that the boundary of a jet preserves $5 \%$ of the velocity on the center line. However, the same angle along a level plane $2.5 \mathrm{~mm}$ above the floor is $20^{\circ}$ because

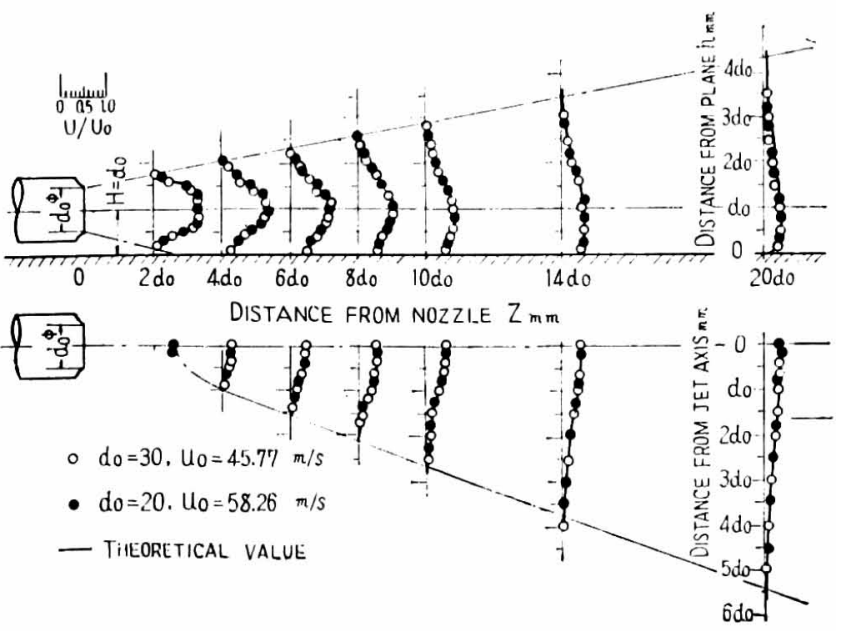

Fig. 19 Experimental and theoretical values of velocity on a circular jet parallel to the plane 
of the effects the floor. The distribution of the velocity ratio $u_{b} / u_{0}$ on a plane $2.5 \mathrm{~mm}$ above the

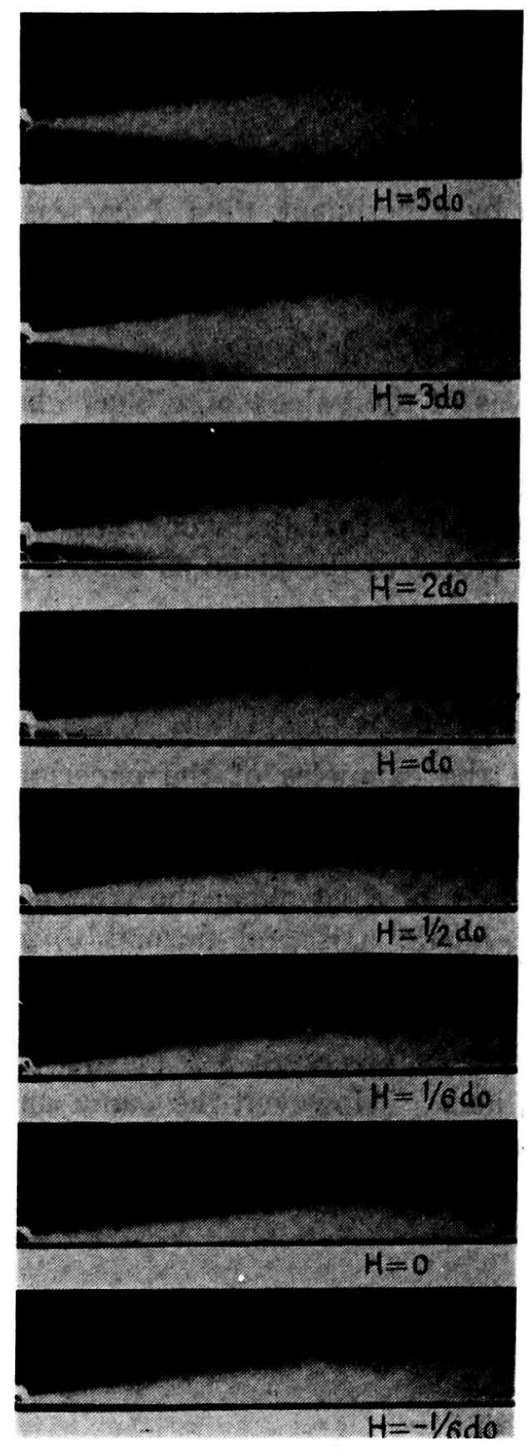

Fig. 20 Aspect of a jet parallel to the plane

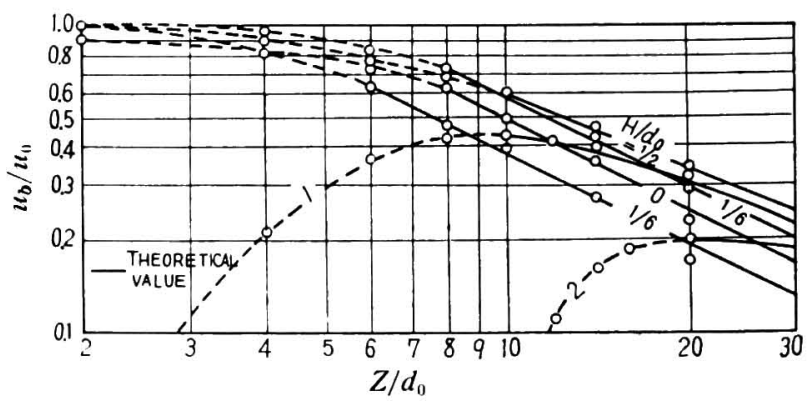

Fig. 21 Distribution of velocity ratio $u_{b} / u_{0}$ on a plane 2.5 $\mathrm{mm}$ high floor is shown in the Fig. 21. For cleaning by blowing, it is desirable that the value of $u_{b} / u_{0}$ be as large as possible and the decline of the ratio mild. Fig. 21, again, suggests that it is better to place the nozzle $d_{0} / 2$ above the floor.

\section{Efficacy of Blow Cleaner in Spinning Mills}

Spinning mills have to remove fly, dust and the like in all processes. Mechanical cleaners, such as travelling fans, have been used lately in some spinning processes, because of the large number and variety of machines used and the large amount of fly which produces broken threads and lowering of yarn quality. Another reason for the use of mechanical cleaners is the saving of manpower.

However, merely turning large-output fans powerfully on spinning machines will not do. It will only have the effect of scattering cotton tufts and landing them on the yarns. We had better blow down cotton tufts from the machines with a minimum of power.

With this in mind, we have designed two types of cleaning apparatus with the cooperation of Nihon Spindle Mfg. Co., Ltd. and investigated their practical characteristics and performance in some mills where these types of apparatus are used. The apparatus is called "Blow Cleaner" and is of types D and R.

Type D (Fig. 22) operates as follows: Exhaust air from "Pneumaniez"[5] is led into the upper part of the spinning machines through a duct, and is blown into the blow tube which is fixed to the runner on the duct and dangles on two sides of each spinning machine. Air is then discharged through outlets bored on the tube to the places needing cleaning.

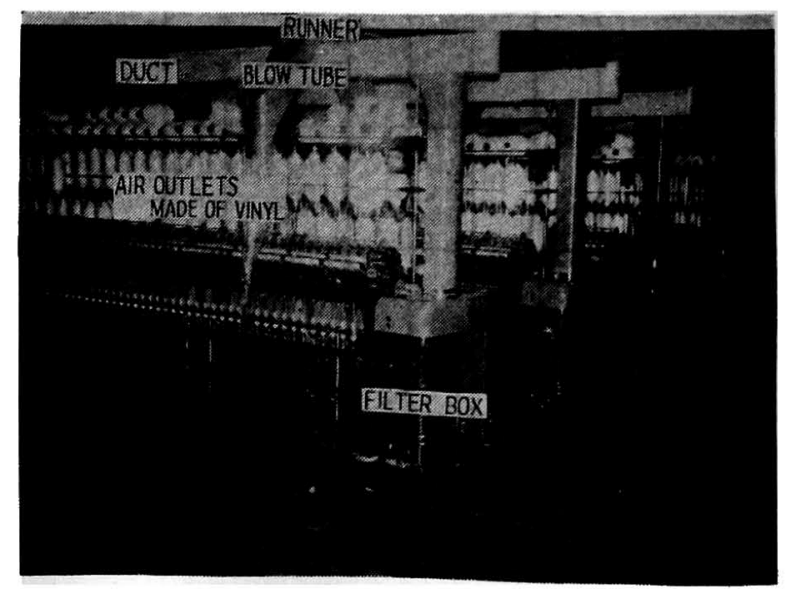

Fig. 22 Blow cleaner, type D 
Type $R$ (Fig. 23) has a motor-equipped runner which runs by its own power on the rails built on the spinning machines. Air is blown from the runner to both sides of the machines.

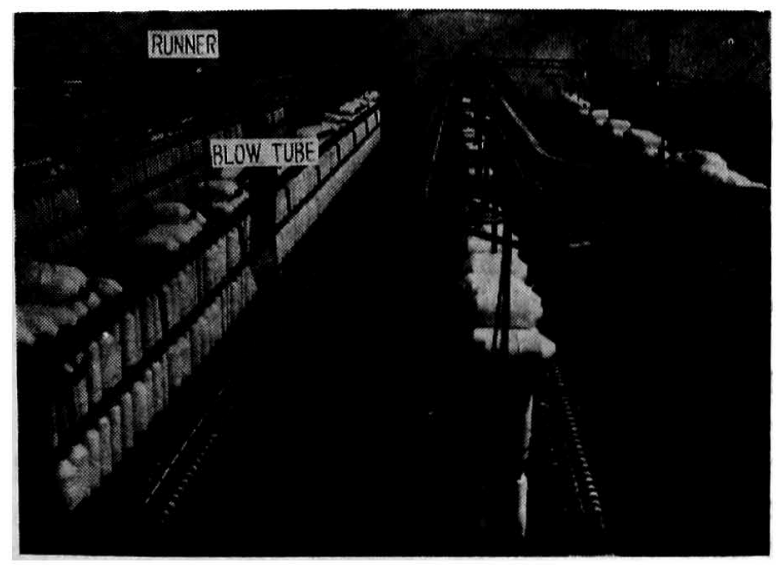

Fig. 23 Blow cleaner, type $\mathbf{R}$

Fig. 24 shows the distribution of air velocity from the outlets of type $D$, the flow rate being $4.65 \mathrm{~m}^{3} / \mathrm{min}$. Fig. 25 refers to type $R$, the flow rate being $4.11 \mathrm{~m}^{3} / \mathrm{min}$. The values of jet velocity $u_{0} \mathrm{~m} / \mathrm{s}$ at all outlets are about equal, being $16.5 \mathrm{~m} / \mathrm{s}$ for type $\mathrm{D}$ and $12 \mathrm{~m} / \mathrm{s}$ for type $\mathrm{R}$. The distribution of the velocity ratio $u_{c} / u_{0}$ on the axis is parallel to

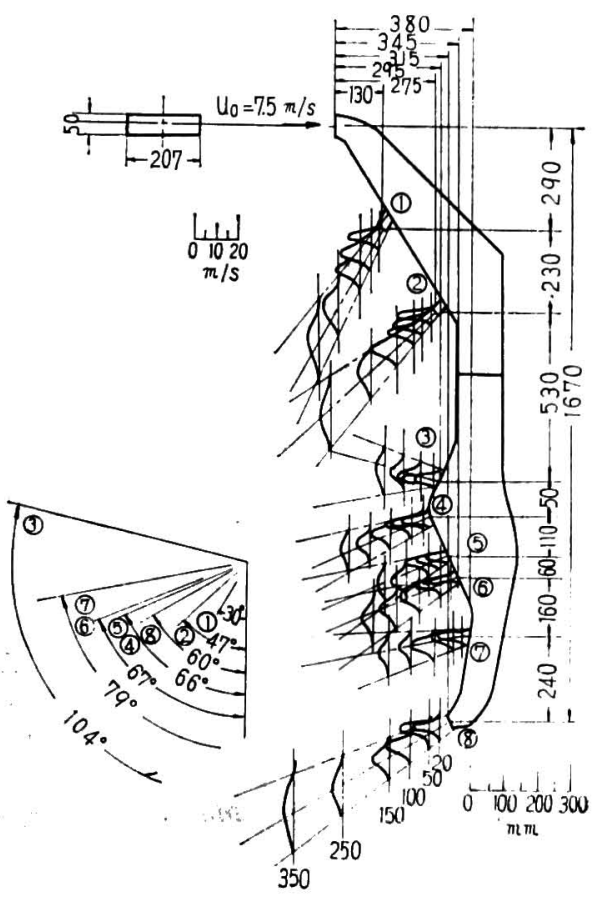

Fig. 24 Velocity distribution of air discharged from the outlets of type D

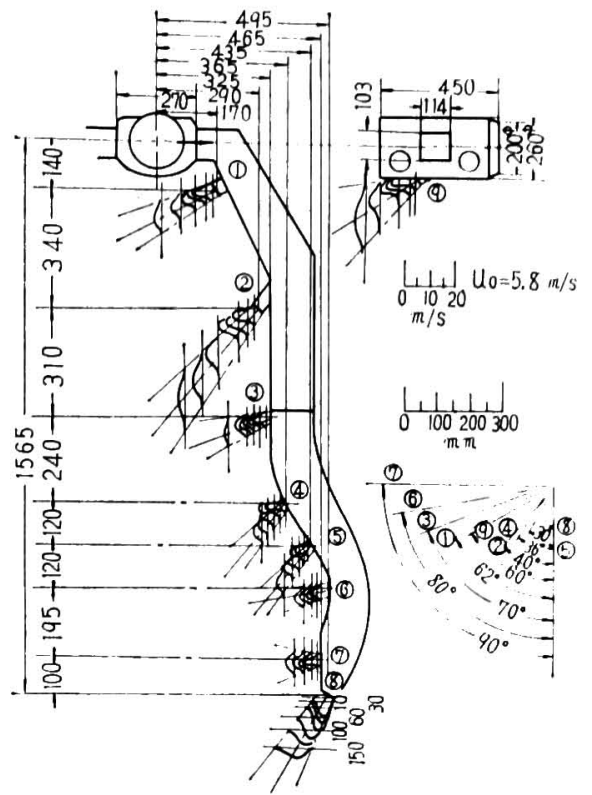

Fig. 25 Velocity distribution of air discharged from the outlets of type $R$

those of a free circular jet issuing from the standard nozzle i.e., a line in Fig. 13, in the regions far from the source, $z / a \geqq 10$. Here $z$ is the distance from the source, $a$ is the radius of a circular outlet, and $a$ is one-half the minor axis for an elliptic outlet and one-half the minor side for a rectangular outlet. The approximate value of $u_{c} / u_{0}$ obtained from experimental formulas are:

\begin{tabular}{|c|c|c|c|c|}
\hline \multicolumn{5}{|c|}{ Values of $A$} \\
\hline Outlet No. & $1 \sim 2$ & $3 \sim 7$ & 8 & 9 \\
\hline Type D & 8.08 & 3.73 & 4.95 & - \\
\hline Type $R$ & 9.48 & 3.73 & 6.23 & 7.53 \\
\hline
\end{tabular}

( $A=10$ for a free circular jet from a standard nozzle)

Figs. 26 and 27 show the distribution of the flow rate $Q \mathrm{~m}^{3} / \mathrm{min}$ discharged from each outlet. On type $D$, the total of the flow rate, $Q_{0} \mathrm{~m}^{3} / \mathrm{min}$., changes according to the position of the runner, the condition of the filter box, etc. However, irrespective of $Q$, the distribution of $Q / Q_{0}$ is the same in any $Q_{0}$, and the rate of flow from the outlets, except outlet No. 8 , is about the same. On type $R$, the rate of flow from the outlets Nos. 3 to 7 is about the same. 
On both types, the flow rate is in proportion to the area of the outlet. Therefore, we can obtain an estimated values of the flow rate by adjusting the outlet area.

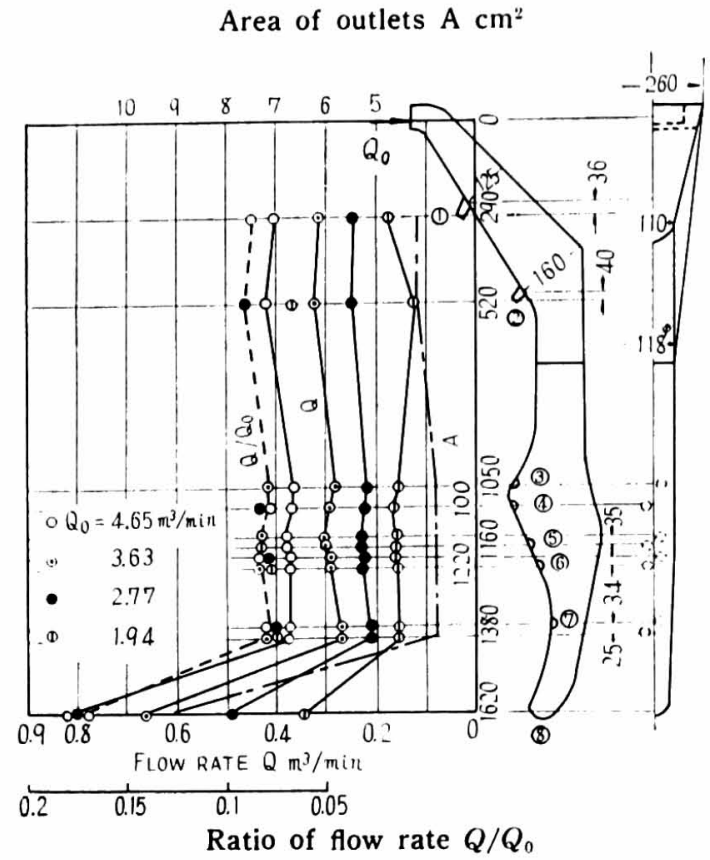

Fig. 26 Distribution of the rate of flow from the outlets of type D

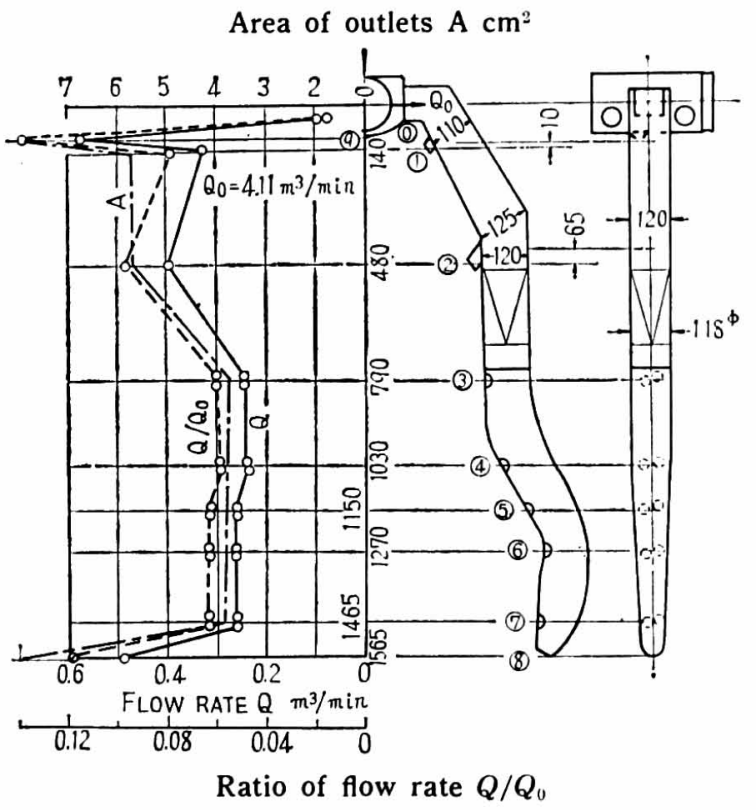

Fig. 27 Distribution of the rate of flow from the outlets of type $\mathbf{R}$
"Blow Cleaner" reduces yarn neps, reduces yarn loss from $15 \%$ to $10 \%$ and saves manpower by one person per bale in a 50,000-spindle mill producing $30 \sim 40$ counts.

\section{Conclusions}

We have looked into the characteristics of a circular jet, normal, inclined and parallel to a plane on the basis of Reichardt's hypothesis, and formulated fluid-dynamical formulas on them. We have measured experimentally the velocity distribution in conditions surrounding the location of each nozzle and obtained the constant $c$ in Reichardt's theory. We have compared the constant with the theoretical values and explained the influences of the plane upon a jet flow. We have obtained the velocity distribution on the plane as an important piece of information on dust cleaning. Finally, we have cited "Blow Cleaner" as an example of a cleaning apparatus for spinning machines and explained its characteristics.

\section{References}

[1] H. Reichardt; "Über eine neue Theoric der freien Turbulenz", Z.A.M.M., 21, 5, 257 (1941)

[2] Y. Niitsu, T. Kato: "Researches on Performance and Design of Air Curtain" Journal of the D.S.E. Japan. 32, 10, 587 (1958)

[3] D. Kuchemann, J Weber; "Aerodynamics of Propulsion" (1953) (McGraw-Hill)

Y. Niitsu, A. Kurahashi, M. Yamasaki; Trans. JSME, 21, 108, 634 (1955)

H. B. Nottage, J.G. Slaby, W. p. Goisza ; H.P.A.C., 123 (1952-7)

W. Tollmien; "Berechung der turbulenten Ausbreitungsvorgange", Z.A.M.M. 6, 468 (1926)

G. L. Tuve; "Air Velocities in Ventilating Jets", H.P.A.C (1953)

[4] A. Koestel, J. B. Austin: "Air Velocities in Two Parallel Ventilating Jets", H.P.A.C., 28, 2, 137 140 (1952)

[5] Y. Niitsu, A. Kurahashi: Text. Mach. Soc., Japan, 5, 10 $(1952-10)$ 